\title{
A METRIC DEFORMATION AND THE FIRST EIGENVALUE OF LAPLACIAN ON 1-FORMS
}

\author{
TAKASHI OTOFUJI
}

\begin{abstract}
We search for a higher-dimensional analogue of Calabi's example of a metric deformation, quoted by Cheeger, which inspired him to prove an inequality between the first eigenvalue of the Laplacian on functions and an isoperimetric constant. We construct an example of a metric deformation on $S^{n}, n \geq 5$, where the first eigenvalue of the Laplacian on functions remains bounded above from zero, and the first eigenvalue of the Laplacian on 1-forms tends to zero. This metric deformation makes the sphere in the limit into a manifold with a cone singularity, which is an intermediate point on a path of deformation from an $\left(S^{n}\right.$, some metric) to an $\left(S^{n-1} \times S^{1}\right.$, some metric).
\end{abstract}

\section{INTRODUCTION}

Calabi constructed the well-known "dumbbell" example of metric deformation on a closed Riemannian manifold such that $\lambda_{0,1}$, the first eigenvalue of the Laplacian acting on smooth functions, goes to zero. Cheeger [Che], suggested by that example, gave a lower bound for $\lambda_{0,1}$ by an isoperimetric constant:

$$
\lambda_{0,1} \geq(1 / 4) h(M)^{2},
$$

where $M$ is an $n$-dimensional closed Riemannian manifold, and

$h(M)=\inf \left\{\frac{\operatorname{vol}_{n-1}(\partial D)}{\operatorname{vol}_{n}(D)}: D\right.$ is a domain of $M$

with smooth boundary such that $\left.\operatorname{vol}_{n}(D) \leq(1 / 2) \operatorname{vol}_{n}(M)\right\}$.

This result indicates that a deformation of a Riemannian metric on a closed manifold in which $\lambda_{0,1} \rightarrow 0$ ends with a break of the manifold, i.e., the number of the connected components $=\operatorname{rank} H^{0}(M, \mathbf{R})$ jumps up. (Of course we must confine ourselves to the cases under some normalizing conditions, for example, boundedness of the diameter, etc.) We ask the question whether there exist higher-dimensional phenomena analogous to the above, in other words, whether metric deformations, with $\lambda_{k, 1}$, the first nonzero eigenvalue of the Laplacian on $k$-forms, tending to zero, make rank $H^{k}(M, \mathbf{R})$ jump up in the limit. Dodziuk posed this question in [Cha, p. 342], conjecturing the existence

Received by the editors November 13, 1989 and, in revised form, June 3, 1991.

1991 Mathematics Subject Classification. Primary 58G25.

Key words and phrases. Metric deformation, Laplacian, 1-form, first eigenvalue, isoperimetric constant. 
of some higher codimensional isoperimetric constant. At present, we have little hope that a general answer may soon be found. Therefore, we search for a higher-dimensional version of Calabi's example.

In the present paper we construct an example of metric deformation in which $\lambda_{1,1}$, the first nonzero eigenvalue of the Laplacian acting on smooth 1 -forms, tends to zero. It is constructed on the sphere $S^{n}, n \geq 5$, where $S^{n}$ converges in the sense of Hausdorff convergence to an $n$-dimensional Riemannian manifold with a cone singularity, and $\lambda_{0,1}$ remains bounded above from zero. The precise statement of the spectral properties of this metric deformation is given in $\S 2$, Theorem 2.1. Now we outline the idea of the construction. First, we collapse $\{\mathbf{0}\} \times S^{1}$ in $S^{n-1} \times S^{1}$ to a point $\mathbf{p}$ and introduce a metric on it so that near $\mathbf{p}$ it is isometric to a metric cone $C$ of $S^{n-2} \times S^{1}$. Then we remove a neighborhood of $\mathbf{p}$, which is a small cone homothetic to $C$, and attach suitably a small $S^{n-2} \times D^{2}$ along the boundaries. From the differentiable viewpoint, this operation is equivalent to the surgery procedure of obtaining $S^{n}$ from $S^{n-1} \times S^{1}$ by removing $D^{n-1} \times S^{1}$ and attaching suitably $S^{n-2} \times D^{2}$. In the metric space of metric spaces with Hausdoff distance, the limit Riemannian manifold is on both the boundary of the set of Riemannian manifolds diffeomorphic to $S^{n}$ and the boundary of the set of Riemannian manifolds diffeomorphic to $S^{n-1} \times S^{1}$.

Remark. For the relations between the convergence of manifolds and spectra, we refer the reader to, for example, Anné [A], Colbois-Courtois [C-C 1, 2] Chavel-Feldman [Cha-F 1, 2], and Fukaya [F]. Colbois-Courtois [C-C 1] treats the case of $p$-forms and shows that Cheeger's constant does not permit to control the first nonzero eigenvalue of the $p$-spectrum.

Plan. In $\S 2$, we explain the construction of the metric deformation and some of its properties. In $\S 3$, we show, assuming Proposition 3.2, that the deformation has the desired property of $\lambda_{0,1}$. In $\S 4$, we prove Proposition 3.2. For the purpose, we investigate the behavior of geodesics on general metric cones.

Acknowledgment. The author would like to thank the referee for suggesting improvements of the presentation and indicating many references. He is also grateful to Professor S. Tanno and H. Muto for their enthusiastic encouragement and valuable advices during the work.

\section{Notations}

Here, let $M$ denote any Riemannian manifold, $g$ the Riemannian metric on $M$, and let $\operatorname{dim} M=n$.

(1) Sobolev isoperimetric constant $\operatorname{Sob}(M)$ :

$\operatorname{Sob}(M)=\inf \left\{\frac{\{A(\partial \Omega)\}^{n}}{\{V(\Omega)\}^{n-1}} ; \Omega\right.$ is a domain of $M$ with smooth boundary and with compact closure $\}$.

where $V$ denotes the $n$-dimensional measure and $A$ the $(n-1)$-dimensional measure induced by the Riemannian metric on $M$.

(2) $U_{x} M=\left\{\xi \in T_{x} M ;|\xi|=1\right\}, \mu$ : the canonical measure on $U_{x} M$ induced by the Riemannian tensor $g_{x}$ on $T_{x} M$. 
(3) Metric cones of $M$ :

$$
C(M)=\left(\mathbf{R}^{+} \times M, d r^{2}+r^{2} g\right),
$$

where $r \in(0, \infty)$ the canonical coordinate of $\mathbf{R}^{+}$. Furthermore,

$$
\begin{aligned}
C_{a, b}(M)=\{(r, x) \in C(M) ; a & <r<b\}, \\
\pi: C(M) & \rightarrow M:(r, x) \mapsto x, \quad \tilde{\pi}: C(M) \rightarrow \mathbf{R}^{+}:(r, x) \mapsto r .
\end{aligned}
$$

(4) $H_{0}^{1,2}(M)=$ the completion of $C_{0}^{\infty}(M)$ with respect to the norm $\|f\|=$ $\left(\int_{M}\left(|f|^{2}+|d f|^{2}\right)\right)^{1 / 2}$.

(5) $g_{s^{n}}$ denotes the Riemannian metric of the standard unit $n$-sphere. In $S^{1}, s$ denotes a unit parameter, and so we have $d s^{2}(s)=g_{s^{1}}(s)$.

(6) Without particular mention, geodesics have unit speed.

(7) dist $_{M}$ is the distance on $M$ induced by the Riemannian metric $g$.

(8) $\operatorname{inj}(M)$ is the injectivity radius of $M$.

\section{AN EXAMPLE OF METRIC DEFORMATION ON $S^{n}, n \geq 5$}

In this section we construct a family of Riemannian metrics $g_{\varepsilon}, \varepsilon>0$, on $S^{n}, n \geq 5$, which is roughly described in the Introduction, and see that it has the properties (1),(2) below. The fact that it has the property (3) will be proved in $\S 3$.

Theorem 2.1. There exists a family of Riemannian metrics $g_{\varepsilon}, \varepsilon>0$, on $S^{n}, n \geq 5$, which has the following properties:

(1) When $\varepsilon \rightarrow 0,\left(S^{n}, g_{\varepsilon}\right) \rightarrow M$ in the sense of Hausdorff convergence where $M$ is the interior of an n-dimensional Riemannian manifold with a cone singularity.

(2) $\lambda_{1,1}^{\prime \prime}(\varepsilon) \rightarrow 0$ as $\varepsilon \rightarrow 0$, where $\lambda_{1,1}^{\prime \prime}(\varepsilon)$ is the first nonzero eigenvalue of the Laplacian on coclosed 1-forms on $\left(S^{n}, g_{\varepsilon}\right)$.

(3) We denote by $\bar{\Delta}_{D}$ the selfadjoint extension of the Laplacian defined on $C_{0}^{\infty}(M)$, whose domain is included in $H_{0}^{1,2}(M)$. Then,

(A) All the spectra of $\bar{\Delta}_{D}$ are eigenvalues and we have: $0=\lambda_{0}<$ $\lambda_{1} \leq \lambda_{2} \leq \cdots \rightarrow \infty$.

(B) When we denote by $\sigma_{i}(\varepsilon)$ the ith eigenvalue of the Laplacian on functions on $\left(S^{n}, g_{\varepsilon}\right)$, then $\sigma_{i}(\varepsilon) \rightarrow \lambda_{i}$ as $\varepsilon \rightarrow 0$ for each $i$.

From now on we denote the Riemannian manifold $\left(S^{n}, g_{\varepsilon}\right)$ by $M_{\varepsilon}$.

Construction of $M, M_{\varepsilon}$. We construct $M$ first, and then modify it into $M_{\varepsilon}$.

(i) Introduce a metric $\tilde{g}$ on $S^{n-1}$, such that $D^{n-1}(2)=\left\{x \in \mathbf{R}^{n-1} ;\|x\|<\right.$ $2\}$, is embedded isometrically somewhere in $\left(S^{n-1}, \tilde{g}\right)$. Let $\mathbf{0}$ be the origin of $D^{n-1}(2)$, and consider it as a point on $S^{n-1}$.

(ii) Next define a metric $g$ on the product $\left(S^{n-1} \backslash\{\mathbf{0}\}\right) \times S^{1}$ by

$$
g_{(x, s)}=\tilde{g}(x)+\{f(x)\}^{2} d s^{2}, \quad \text { where }(x, s) \in\left(S^{n-1} \backslash\{\mathbf{0}\}\right) \times S^{1},
$$

where $f(x)$ satisfies

$$
\begin{aligned}
& f(x) \in C^{\infty}\left(S^{n-1} \backslash\{\mathbf{0}\}\right), \quad f>0, \\
& f(x)=\operatorname{dist}_{\left(S^{n-1}, \tilde{g}\right)}(\mathbf{0}, x) \quad \text { on } D^{n-1}(2) \backslash\{\mathbf{0}\}\left(\subset S^{n-1}\right) .
\end{aligned}
$$


We denote by $M$ the Riemannian manifold $\left(\left(S^{n-1} \backslash\{\mathbf{0}\}\right) \times S^{1}, g\right)$.

(iii) We see that $\left(D^{n-1}(2) \backslash\{\mathbf{0}\}\right) \times S^{1}$ (as a Riemannian submanifold of $M$ ) and $C_{0,2}\left(S^{n-2} \times S^{1}\right)$ are isometric under the following map:

$$
\left(D^{n-1}(2) \backslash\{\mathbf{0}\}\right) \times S^{1} \ni((r, \theta), s) \mapsto(r,(\theta, s)) \in C_{0,2}\left(S^{n-2} \times S^{1}\right),
$$

where $(r, \theta)$ is the polar coordinate of $D^{n-1}(2) \backslash\{\mathbf{0}\}, r \in(0,2), \theta \in S^{n-2}$. Therfore $M$ is the interior of a Riemannian manifold with a cone singularity.

(iv) Construct $M_{\varepsilon}$ by modifying $M$ near the singular point. First, for $\varepsilon>0$, introduce a metric $h_{\varepsilon}$ on $S^{n-2} \times \bar{D}_{\varepsilon}^{2}$ in the following way:

(1) Introduce the polar coordinate $(r, s), 0 \leq r \leq \varepsilon, s \in S^{1}$, on $\bar{D}_{\varepsilon}^{2}$.

(2) Take a $C^{\infty}$-function $\alpha(r)$ on $[0,1]$ which satisfies

$$
\alpha(r)= \begin{cases}\frac{2}{3} & \text { if } 0 \leq r \leq \frac{1}{2} \\ r & \text { if } \frac{3}{4} \leq r \leq 1\end{cases}
$$

and define $\alpha_{\varepsilon}(r)=\varepsilon \alpha(r / \varepsilon)$.

(3) Define a metric $h_{\varepsilon}$ on $S^{n-2} \times \bar{D}_{\varepsilon}^{2}$ as

$$
h_{\varepsilon}(\theta,(r, s))=\left(\alpha_{\varepsilon}(r)\right)^{2} g_{s^{n-2}}(\theta)+d r^{2}+r^{2} g_{s^{1}}(s) \text {. }
$$

We denote the Riemannian manifold $\left(S^{n-2} \times \bar{D}_{\varepsilon}^{2}, h_{\varepsilon}\right)$ by $H_{\varepsilon}$. We remark that $H_{\varepsilon}$ is homothetic to $H_{1}$.

(v) Glue $H_{\varepsilon}$ and $M \backslash C_{0, \varepsilon}\left(S^{n-2} \times S^{1}\right)$ along their boundaries under the map:

$$
\partial\left(S^{n-2} \times \bar{D}^{2}\right) \ni(\theta,(\varepsilon, s)) \mapsto(\varepsilon,(\theta, s)) \in \partial\left(M \backslash C_{0, \varepsilon}\left(S^{n-2} \times S^{1}\right)\right) .
$$

From the expressions of the metrics by the polar coordinates it is easy to see that the glued metric is smooth. We denote by $M_{\varepsilon}$ the glued Riemannian manifold $H_{\varepsilon} \cup\left(M \backslash C_{0, \varepsilon}\left(S^{n-2} \times S^{1}\right)\right)$.

(vi) It is easy to see that $M_{\varepsilon}$ is diffeomorphic to $S^{n}$, and that $M_{\varepsilon}$ converges to $M$ in the sense of Hausdorff convergence.

We check that the family $M_{\varepsilon}, \varepsilon>0$, has the property (2):

Proposition 2.2. $\lambda_{1,1}^{\prime \prime}(\varepsilon) \rightarrow 0$ as $\varepsilon \rightarrow 0$.

Lemma 2.3. The 1-form $d s$ is closed and coclosed on the Riemannian manifold $\left(N \times S^{1}, g+\beta^{2} g_{S^{1}}\right)$, where $(N, g)$ is a Riemannian manifold and $\beta \in$ $C^{\infty}(N), \beta>0$. (ds is the pull-back of the canonical 1-form on $S^{1}$.)

Proof. It is obvious that $d(d s)=0$. We show $d * d s=0$. Let $\omega_{N}$ the volume form of $N$. Then, since $|d s|=\beta^{-1}, * d s=\beta(x)^{-1} \omega_{N}$. Therefore $d(* d s)=$ $\frac{\partial}{\partial s}\left(\beta(x)^{-1}\right) d s \wedge \omega_{N}+(\beta(x))^{-1} d \omega_{N}=0$. Q.E.D.

Proof of Proposition 2.2. There is no nonzero harmonic 1-form on $M_{\varepsilon}$, since $H^{1}\left(M_{\varepsilon}, \mathbf{R}\right)=0$. So, it is enough to find a 1 -form $\omega_{\varepsilon}$ on $M_{\varepsilon}$, for each $\varepsilon>0$, whose Rayleigh quotient $R_{\varepsilon}$ goes to zero when $\varepsilon \rightarrow 0$. Let

$$
\check{H}_{\varepsilon}=\left\{(\theta,(r, s)) \in H_{\varepsilon} ; 0 \leq r \leq \frac{\varepsilon}{2}\right\} .
$$

$M_{\varepsilon} \backslash \check{H}_{\varepsilon}$ is isomorphic to the Riemannian manifold $\left(S^{n-1} \backslash D^{n-1}\left(\frac{\varepsilon}{2}\right)\right) \times S^{1}$ with metric $\tilde{g}+\kappa^{2}(x) d s^{2}$, where $\kappa \in C^{\infty}\left(S^{n-1} \backslash D^{n-1}\left(\frac{\varepsilon}{2}\right)\right)$ is defined as

$$
\kappa(x)= \begin{cases}f(x) & \text { if } x \in S^{n-1} \backslash D^{n-1}(\varepsilon), \\ \alpha(x) & \text { if } x=(r, \theta) \in D^{n-1}(\varepsilon) \backslash D^{n-1}\left(\frac{\varepsilon}{2}\right) .\end{cases}
$$


( $f$ is introduced in the construction of $M$, and $\alpha$ in the construction of $H_{\varepsilon}$.) On $M_{\varepsilon} \backslash \breve{H}_{\varepsilon}$ we define $\omega_{\varepsilon}$ by $\omega_{\varepsilon}=d s$. By Lemma 2.3 , this form is closed and coclosed. On $\check{H}_{\varepsilon}$ we define $\omega_{\varepsilon}$ by $\omega_{\varepsilon}=\frac{r^{2}}{\varepsilon^{2}} d s .((r, s)$ is the polar coordinate used in the construction of $H_{\varepsilon}$.) The 1 -form $\omega_{\varepsilon}$ on $M_{\varepsilon}$ has the following properties:

- There exists a positive constant $c$ such that for any $\varepsilon>0, \int_{M_{\varepsilon}}\left|\omega_{\varepsilon}\right|^{2}>\varepsilon$.

- $\partial \omega_{\varepsilon}=0$ on $M_{\varepsilon}$.

○ $d \omega_{\varepsilon}=0$ on $M_{\varepsilon} \backslash \check{H}_{\varepsilon}$.

- $\left|d \omega_{\varepsilon}\right|=\operatorname{const} \varepsilon^{-2}$ on $\check{H}_{\varepsilon}$ and $\operatorname{vol}\left(\check{H}_{\varepsilon}\right)=\varepsilon^{n} \operatorname{vol}\left(\check{H}_{1}\right)$, so we have

$$
\begin{aligned}
\int_{\check{H}_{\varepsilon}}\left|d \omega_{\varepsilon}\right|^{2} & =\operatorname{const}\left(\varepsilon^{-2}\right)^{2} \times \varepsilon^{n} \operatorname{vol}\left(\check{H}_{1}\right) \\
& =\operatorname{const} \varepsilon^{n-4} \rightarrow 0 \text { as } \varepsilon \rightarrow 0 .
\end{aligned}
$$

Therefore $R_{\varepsilon} \rightarrow 0$ as $\varepsilon \rightarrow 0$.

Remark. By the homothety of the $H_{\varepsilon}$ 's, the curvature of $M_{\varepsilon}$ is not bounded under the deformation. The condition $n=\operatorname{dim} M_{\varepsilon} \geq 5$ is crucial in the last step of the proof of Proposition 2.2. The author does not know whether a similar example can be constructed in dimensions 3 and 4 .

\section{CoNVERgence of the eigenvalues of the Laplacian on functions}

In this section we check that $M_{\varepsilon}, \varepsilon>0$, have the property (3) stated in $\S 2$. The method of showing the convergence of the heat kernels and the eigenvalues are modeled on that used in Chavel and Feldman [1, 2].

Notations : $q_{\varepsilon}(x, y, t)$ : the Dirichlet heat kernel on $M \backslash C_{0, \varepsilon}$. $p_{\varepsilon}(x, y, t)$ : the heat kernel on $M_{\varepsilon}$.

$\lambda_{i}(\varepsilon)$ : the $i$ th Dirichlet eigenvalue of $M \backslash C_{0, \varepsilon}(i=1,2, \ldots)$. $\sigma_{i}(\varepsilon)$ : the $i$ th eigenvalue of $M_{\varepsilon}(i=0,1, \ldots)$.

Definition. We define $p(x, y, t)$, a function on $M \times M \times(0, \infty)$, as

$$
p(x, y, t)=\sup _{\varepsilon} q_{\varepsilon}(x, y, t) .
$$

Proposition 3.1. $p, p_{\varepsilon}, q_{\varepsilon}$ have the following properties:

(1) [6, Lemma 3.3] For any $x, y \in M \backslash C_{0, \varepsilon}$ and any $t$, we have

$$
\begin{gathered}
q_{\varepsilon}(x, y, t)<p(x, y, t), \quad q_{\varepsilon}(x, y, t)<p_{\varepsilon}(x, y, t), \\
q_{\varepsilon}(x, y, t)<q_{\varepsilon^{\prime}}(x, y, t) \quad\left(\varepsilon>\varepsilon^{\prime}\right) .
\end{gathered}
$$

(2) [6, Lemma 3.7, the proof of Theorem 3.6] $q_{\varepsilon}(x, y, t)$ converges to $p(x, y, t)$ uniformly on any compact subset of $M \times M \times(0, \infty)$.

(3) [6, Theorem 3.6] $p(x, y, t)$ is of class $C^{\infty}$, and is a heat kernel in the following sense:

For each bounded continuous function $u_{0}$ on $M$ we define $u$ : $M \times$ $[0, \infty) \mapsto \mathbf{R}$ as

$$
u(x, t)= \begin{cases}\int_{M} p(x, y, t) u_{0}(y) d V(y), & t>0 \\ u_{0}(x), & t=0 .\end{cases}
$$


Then $u$ is continuous on $M \times[0, \infty)$ and, it is on $M \times(0, \infty)$, of $C^{2}$ class with respect to $x$, of $C^{1}$ class with respect to $t$, and satisfies $\Delta u+\frac{\partial u}{\partial t}=0$. Moreover, $p(x, y, t)>0, p(x, y, t)=p(y, x, t)$, and $\int_{M} p(x, z, t) p(z, y, s) d V(z)=p(x, y, t+s)$.

(4) [6, Proposition 4.5] $p(x, y, t)$ is the kernel of the semigroup with generator $\bar{\Delta}$.

In order to show the convergence of the eigenvalues, we need upper estimates of the heat kernels $p, p_{\varepsilon}, q_{\varepsilon}$.

Proposition 3.2. We can estimate the Sobolev isoperimetric constant of $H_{\varepsilon} \cup$ $C_{\varepsilon, 1}\left(S^{n-2} \times S^{1}\right), n \geq 5$, for any $\varepsilon>0$ small enough from below by a positive constant independent of $\varepsilon$.

We prove this proposition in $\S 4$.

Proposition 3.3. Suppose $\varepsilon>0$ is small enough. Then, there is a constant $C(\alpha, \beta)$ for any $\alpha, \beta \in(0, \infty), \alpha<\beta$, independent of $\varepsilon$, which satisfies the following: For any $x, y, t \in[\alpha, \beta]$, and $\varepsilon$, we have

$$
p_{\varepsilon}(x, y, t), q_{\varepsilon}(x, y, t), p(x, y, t) \leq C(\alpha, \beta) .
$$

Proof. We see from Proposition 3.1 that it is enough to prove for $p_{\varepsilon}(x, y, t)$. According to [3, p. 198], we have the estimate

$$
p_{\varepsilon}(x, y, t): \text { const }\left\{t^{-\frac{n}{2}}+t r^{-(n+2)}\right\}\left\{\operatorname{Sob}\left(B_{r}(x)\right) \cdot \operatorname{Sob}\left(B_{r}(y)\right)\right\}^{-\frac{1}{2}}
$$

for $x, y \in M_{\varepsilon}$. Thus we only have to bound Sob $B_{\frac{1}{4}}(x)$, the Sobolev isoperimetric constant of $B_{\frac{1}{4}}(x)$ for each $x \in M_{\varepsilon}$ from below. We assume $\varepsilon<\frac{1}{4}$. For $x \in H_{\varepsilon} \cup C_{\varepsilon, \frac{1}{2}}\left(S^{n-2} \times S^{1}\right), B_{\frac{1}{4}}(x)$ is contained in $H_{\varepsilon} \cup C_{\varepsilon, 1}\left(S^{n-2} \times S^{1}\right)$, and so, using Lemma 3.2, we can estimate $\operatorname{Sob}\left(B_{\frac{1}{4}}(x)\right)$ from below by a positive constant independent of $x$ and $\varepsilon$. For $x \in M \backslash C_{0, \frac{1}{2}}\left(S^{n-2} \times S^{1}\right), B_{\frac{1}{4}}(x)$ is contained in $M \backslash C_{0, \frac{1}{4}}\left(S^{n-2} \times S^{1}\right)$, and thus this case is the same as the above.

Proposition 3.4. (1) For each $t>0$,

$$
H_{t}: L^{2}(M) \rightarrow L^{2}(M), \quad f(x) \mapsto \int_{M} p(x, y, t) f(y) d V(y)
$$

is a compact selfadjoint operator.

(2) The eigenvalues of $H_{t}$ are

$$
\exp \left(-\lambda_{0} t\right) \geq \exp \left(-\lambda_{1} t\right) \geq \cdots(\rightarrow 0)
$$

The eigeinfunction corresponding to the eigenvalue $\exp \left(-\lambda_{i} t\right)$ is a function independent of $t$. We denote it by $\varphi_{i}$.

(3) The eigenfunction $\varphi_{i}$ is of class $C^{2}$.

(4) The development

$$
p(x, y, t)=\sum_{i=0}^{\infty} \exp \left(-\lambda_{i} t\right) \varphi_{i}(x) \varphi_{i}(y)
$$

is uniformly convergent on any compact subset of $M \times M$.

Proof. (1) We see that $H_{t}$ is an integral operator of Hilbert-Schmidt type by Proposition 2.3 and by the finiteness of the volume of $M$. 
(2) By (1) and by the fact that $H_{t}$ has semigroup property.

(3) We can see that $\varphi_{i}$ is continuous and bounded from the expression

$$
\exp \left(-\lambda_{i} t\right) \varphi_{i}(x)=\int_{M} p(x, y, t) \varphi_{i}(x) d V(y),
$$

and that it is of class $C^{2}$ from Proposition 3.1(3).

(4) We see that $p(x, y, t)$ is a positive kernel from the fact that $H_{t}$ has semigroup property. We can claim (4) by this fact and the continuity of $p$ using Mercer's theorem (suitably modified to the case of a noncompact domain with finite volume).

Proposition 3.5. (1) All the spectra of $\bar{\Delta}_{D}$ are eigenvalues and we have:

$$
0 \leq \lambda_{0} \leq \lambda_{1} \leq \cdots \rightarrow+\infty .
$$

The corresponding eigenfunctions are the $\varphi_{i}$ 's in Proposition 3.4.

(2) The constant function 1 is an eigenfunction of $\bar{\Delta}_{D}$ corresponding to the eigenvalue 0 . In particular $\lambda_{0}=0$.

(3) $\lambda_{0}<\lambda_{1}$.

Proof. (1) $\exp \left(-\bar{\Delta}_{D} t\right)=H_{t}$ and Proposition 3.4(2) imply all except for $0 \leq \lambda_{0}$. The claim $0 \leq \lambda_{0}$ can be verified by the formula $\left\langle\bar{\Delta}_{D} \varphi, \varphi\right\rangle_{L^{2}}=\left\langle\bar{d}_{c} \varphi, \bar{d}_{c} \varphi\right\rangle_{L^{2}}$, since $\varphi \in \operatorname{Dom}\left(\bar{\Delta}_{D}\right)$, where $\bar{d}_{c}$ is the closure of the exterior differential operator $d$ operating on $C_{0}^{\infty}(M)$.

(2) First we show $1 \in H_{0}^{1,2}(M)$. We approximate 1 by elements of $C_{0}^{\infty}(M)$ as follows. We define $h_{\varepsilon} \in C_{0}^{\infty}(M)$ by

$$
\begin{aligned}
h_{\varepsilon}(x) & =1, & & x \in M \backslash C_{0, \varepsilon}\left(S^{n-2} \times S^{1}\right), \\
h_{\varepsilon}(r, x) & =\varphi(r / \varepsilon), & & (r, x) \in C_{0, \varepsilon}\left(S^{n-2} \times S^{1}\right), r \in(0, \varepsilon], \quad x \in S^{n-2} \times S^{1},
\end{aligned}
$$

where $\varphi \in C^{\infty}((0, \infty))$ satisfies $0 \leq \varphi(r) \leq 1, \varphi=0$ on $\left(0, \frac{1}{2}\right], \varphi=1$ on $[1, \infty)$. Then we have $h_{\varepsilon} \rightarrow 1$ as $\varepsilon \rightarrow 0$ in $L^{2}$. And $\left|d h_{\varepsilon}\right|=$ const $\varepsilon^{-1}$ on $C_{0, \varepsilon}\left(S^{n-2} \times S^{1}\right)$, and $\operatorname{vol}\left(C_{0, \varepsilon}\left(S^{n-2} \times S^{1}\right)\right)=$ const $\varepsilon^{-5}$ implies

$$
\int_{M}\left|d h_{\varepsilon}-d \cdot 1\right|^{2}=\int_{C_{0, \varepsilon}\left(S^{n-2} \times S^{1}\right)}\left|d h_{\varepsilon}\right|^{2}=\text { const } \varepsilon^{n-2},
$$

from which we obtain $d h_{\varepsilon} \rightarrow d \cdot 1=0$ as $\varepsilon \rightarrow 0$ in $L^{2}$. Therefore we have $1 \in H_{0}^{1,2}(M)$. And we see $1 \in \operatorname{Dom}\left(\bar{\Delta}_{D}\right), \bar{\Delta}_{D} 1=0$ from the fact that the map $C_{0}^{\infty}(M) \ni \varphi \mapsto\langle\Delta \varphi, 1\rangle_{L^{2}}\left(=\int_{M} \Delta \varphi=0\right)$ is continuous in $L^{2}$.

(3) Let $\varphi$ be an eigenfunction corresponding to the eigenvalue 0 . Since $0=\left\langle\bar{\Delta}_{D} \varphi, \varphi\right\rangle=\left\langle\bar{d}_{c} \varphi, \bar{d}_{c} \varphi\right\rangle$, we have $\bar{d}_{c} \varphi=0$. But $\varphi$ is of $C^{2}$ class, and thus we have $d \varphi=\bar{d}_{c} \varphi$. Thus we have $d \varphi=0$, which implies that $\varphi$ is constant by the connectedness of $M$.

Proposition 3.6. (1) For each $x \in M$, and each $t>0$, we have

$$
\int_{M} p(x, y, t) d V(y)=1 \text {. }
$$

(2) For each $x \in M$, and each $t>0$, we have

$$
\int_{M} q_{\varepsilon}(x, y, t) d V(y) \rightarrow \int_{M} p(x, y, t) d V(y)=1 .
$$


Proof. (1) The constant function 1 is the eigenfunction of $H_{t}$ corresponding to the eigenvalue $\exp (-0 \cdot t)=1$ (Proposition 3.5(2)).

(2) We can see the convergence by Proposition 3.1(2) and the uniform boundedness of $p$ and $q_{\varepsilon}$.

Proposition 3.7.

$$
\begin{gathered}
\lim _{\varepsilon \rightarrow 0} \int_{M \backslash C_{0, \ell}\left(S^{n-2} \times S^{1}\right)} q_{\varepsilon}(x, x, t) d V(x)=\int_{M} p(x, x, t) d V(x) . \\
\lim _{\varepsilon \rightarrow 0} \int_{M_{\varepsilon}} p_{\varepsilon}(z, z, t) d V(z)=\int_{M} p(x, x, t) d V(x) .
\end{gathered}
$$

Proof. (1) Because, as $\varepsilon \rightarrow 0, q_{\varepsilon}(x, y, t)$ increases monotonically and converges to $p(x, y, t)$.

(2) Since we have

$$
\begin{aligned}
\int_{M_{\varepsilon}} & p_{\varepsilon}(z, z, t) d V(z)-\int_{M} p(x, x, t) d V(x) \\
= & \int_{M_{\varepsilon} \backslash H_{\varepsilon}}\left\{p_{\varepsilon}(x, x, t)-p(x, x, t)\right\} d V(x) \\
& +\int_{H_{\varepsilon}} p_{\varepsilon}(z, z, t) d V(z)-\int_{C_{0, \varepsilon}\left(S^{n-2} \times S^{1}\right)} p(x, x, t) d V(x),
\end{aligned}
$$

it suffices to show that each term of the above formula converges to 0 as $\varepsilon \rightarrow$ 0 . For the second and the third terms, we can verify the convergence by the facts that $p_{\varepsilon}$ and $p$ are bounded uniformly with respect to $\varepsilon$ and that the volumes of the domains of integral converge to 0 as $\varepsilon \rightarrow 0$. For the first term, it is enough to show that $p_{\varepsilon}(x, x, t)$ converges to $p(x, x, t)$ for each $x \in M$, since, because $p_{\varepsilon}$ and $p$ are bounded uniformly with respect to $\varepsilon$ and $\operatorname{vol}(M)<\infty$, we can verify the convergence of the term using Lebesgue's convergence theorem. And it needs only to show $q_{\varepsilon}(x, y, t) \rightarrow p_{\varepsilon}(x, y, t) \rightarrow 0$ as $\varepsilon \rightarrow 0$, since we have $q_{\varepsilon}(x, y, t) \rightarrow p(x, y, t)$. For the purpose, it is sufficient to show $\lim _{\varepsilon \rightarrow 0} \int_{M_{\varepsilon}}\left\{p_{\varepsilon}(x, y, t)-q_{\varepsilon}(x, y, t)\right\} f(y) d V(y)=0$ for any $f \in C_{0}^{\infty}(M)$. But we have

$$
\begin{aligned}
& \left|\int_{M_{\varepsilon}}\left\{p_{\varepsilon}(x, y, t)-q_{\varepsilon}(x, y, t)\right\} f(y) d V(y)\right| \\
& \quad \leq \sup _{y \in M}|f(y)| \int_{M_{\varepsilon}}\left\{p_{\varepsilon}(x, y, t)-q_{\varepsilon}(x, y, t)\right\} d V(y) \\
& \quad=\sup _{y \in M}|f(y)|\left\{1-\int_{M} q_{\varepsilon}(x, y, t) d V(y)\right\},
\end{aligned}
$$

which converges to 0 as $\varepsilon \rightarrow 0$ by Proposition 3.6(2).

Proposition 3.8. For each $i=0,1, \ldots$,

$$
\lambda_{i+1}(\varepsilon) \rightarrow \lambda_{i}, \quad \sigma_{i}(\varepsilon) \rightarrow \lambda_{i} \quad \text { as } \varepsilon \rightarrow 0 .
$$


Proof. We have

$$
\begin{gathered}
\int_{M} p(x, x, t) d V(x)=\sum_{i=0}^{\infty} \exp \left(-\lambda_{i} t\right), \\
\int_{M_{\varepsilon}} p_{\varepsilon}(z, z, t) d V(z)=\sum_{i=0}^{\infty} \exp \left(-\sigma_{i}(\varepsilon) t\right), \\
\int_{M \backslash C_{0, \ell}\left(S^{n-2} \times S^{1}\right)} q_{\varepsilon}(x, x, t) d V(x)=\sum_{i=0}^{\infty} \exp \left(-\lambda_{i+1}(\varepsilon) t\right) .
\end{gathered}
$$

Therefore, by Proposition 3.7, we have

$$
\begin{aligned}
\lim _{\varepsilon \rightarrow 0} \sum_{i=0}^{\infty} \exp \left(-\lambda_{i+1}(\varepsilon) t\right) & =\sum_{i=0}^{\infty} \exp \left(-\lambda_{i} t\right), \\
\lim _{\varepsilon \rightarrow 0} \sum_{i=0}^{\infty} \exp \left(-\sigma_{i}(\varepsilon) t\right) & =\sum_{i=0}^{\infty} \exp \left(-\lambda_{i} t\right),
\end{aligned}
$$

but on the other hand, by min-max principle,

$$
\begin{gathered}
\varepsilon_{1}>\varepsilon_{2} \Rightarrow \lambda_{i}\left(\varepsilon_{1}\right)>\lambda_{i}\left(\varepsilon_{2}\right), \\
\lambda_{i+1}(\varepsilon)>\lambda_{i}, \\
\lambda_{i+1}(\varepsilon)>\sigma_{i}(\varepsilon),
\end{gathered}
$$

for all $i=0,1, \ldots$ By (3), (4), we have $\lim _{\varepsilon \rightarrow 0} \lambda_{i+1}(\varepsilon)=\lambda_{i}$ for each $i=$ $0,1, \ldots$, since, if there existed a $k$ such that $\lim _{\varepsilon \rightarrow 0} \lambda_{k+1}(\varepsilon)>\lambda_{k}$, (1) would not hold. And by this result and (5) we see that $\lim _{\sup _{\varepsilon \rightarrow 0}} \sigma_{i}(\varepsilon) \leq \lambda_{i}$, for all $i=0,1, \ldots$. But we have also $\liminf _{\varepsilon \rightarrow 0} \sigma_{i}(\varepsilon) \geq \lambda_{i}$ for all $i=0,1, \ldots$. To see this, we show that if there exists a $k$ such that $\liminf _{\varepsilon \rightarrow 0} \sigma_{k}(\varepsilon)<\lambda_{k}$, this contradicts (2). Take a sequence $\left\{\varepsilon_{l}\right\}_{l=1}^{\infty} \subset \mathbf{R}^{+}$such that $\varepsilon_{l} \rightarrow 0, \sigma_{k}\left(\varepsilon_{l}\right) \rightarrow$ $\liminf _{\varepsilon \rightarrow 0} \sigma_{k}(\varepsilon)<\lambda_{k}$ as $l \rightarrow 0$. Then we have

$$
\begin{aligned}
\sum_{i=0}^{\infty} \exp \left(-\lambda_{i} t\right) & =\lim _{l \rightarrow \infty} \sum_{i=0}^{\infty} \exp \left(-\sigma_{i}\left(\varepsilon_{l}\right) t\right) \\
& >\exp \left(-\lambda_{k} t\right)+\sum_{j \neq k} \exp \left(-\left\{\limsup _{l} \sigma_{j}\left(\varepsilon_{l}\right)\right\} t\right) \\
& \geq \exp \left(-\lambda_{k} t\right)+\sum_{j \neq k} \exp \left(-\lambda_{j} t\right) \\
& =\sum_{i=0}^{\infty} \exp \left(-\lambda_{i} t\right) .
\end{aligned}
$$

Therefore we have $\lim _{\varepsilon \rightarrow 0} \sigma_{i}(\varepsilon)=\lambda_{i}$ for all $i=0,1, \ldots$

\section{A LOWER BOUND FOR THE SOBOLEV ISOPERIMETRIC CONSTANT$$
\text { OF } H_{\varepsilon} \cup C_{\varepsilon, 1}\left(S^{n-2} \times S^{1}\right)
$$

In this section we prove:

Proposition 3.2. For $\varepsilon>0$ small enough we can bound from below the Sobolev constant of $H_{\varepsilon} \cup C_{\varepsilon, 1}\left(S^{n-2} \times S^{1}\right)$ with a positive constant independent of $\varepsilon$. 
In 1 we study the behavior of geodesics on metric cones. In 2 we prove Proposition 3.2.

1. On the behavior of geodesics on metric cones. First using coordinates we express the equation of geodesics on a metric cone $C(N)$ where $N$ is an $n$ dimensional Riemannian manifold $(N, g)$. Let $U$ be a coordinate neighborhood of $N,\left(x^{1}, \ldots, x^{n}\right)$ coordinates of $U,\left(g_{i j}\right)$ the coordinate expression of metric $g, \Gamma_{i j}^{k}$ the Christoffel symbol of $\left(g_{i j}\right)$, and $\left(x^{0}\right)$ the standard coordinate of $\mathbf{R}^{+}$. Then we have $U \times \mathbf{R}^{+}$as a coordinate neighborhood in $C(N)$ with coordinates $\left(x^{0}, \ldots, x^{n}\right)$. Let $\left(\tilde{g}_{\alpha \beta}\right)$ be the expression of the metric $\tilde{g}$ of $C(N)$, and $\tilde{\Gamma}_{\alpha \beta}^{\gamma}$ the Christoffel symbol of $\left(\tilde{g}_{\alpha \beta}\right)$. Then we can express $\tilde{g}_{\alpha \beta}$ as

$$
\tilde{g}_{\alpha \beta}= \begin{cases}1, & \alpha=\beta=0, \\ 0, & \alpha=0, \quad \beta \neq 0 \text { or } \alpha \neq 0, \quad \beta=0, \\ \left(x^{0}\right)^{2} g_{\alpha \beta}, & 1 \leq \alpha, \beta \leq n,\end{cases}
$$

and $\tilde{\Gamma}_{\alpha \beta}^{\gamma}$ as:

$$
\begin{array}{cl} 
& \widetilde{\Gamma}_{i j}^{k}=\Gamma_{i j}^{k} \quad(1 \leq i, j, k \leq n), \\
\widetilde{\Gamma}_{i j}^{0}=-x^{0} g_{i j}, \quad & \widetilde{\Gamma}_{0 j}^{0}=\left(x^{0}\right)^{-1} \delta_{i}^{j}, \quad \widetilde{\Gamma}_{0 j}^{0}=\widetilde{\Gamma}_{00}^{k}=\widetilde{\Gamma}_{00}^{0}=0 .
\end{array}
$$

Using the coordinate expression above we can easily see that the equation of a geodesic $\gamma(t)=\left(\gamma^{0}(t)=\tilde{\pi} \circ \gamma(t), \ldots, \gamma^{n}(t)\right)$ in $C(N)$ is expressed with the coordinates $\left(x^{0}, \ldots, x^{n}\right)$ as

$$
\begin{aligned}
\frac{d^{2} \gamma^{0}}{d t^{2}}(t) & -\gamma^{0}(t) \sum_{i, j=1}^{n} g_{i j}(\pi \circ \gamma(t)) \frac{d \gamma^{i}}{d t}(t) \frac{d \gamma^{j}}{d t}(t)=0, \\
\frac{d^{2} \gamma^{k}}{d t^{2}}(t) & +\sum_{i, j=1}^{n} \Gamma_{i j}^{k}(\pi \circ \gamma(t)) \frac{d \gamma^{i}}{d t}(t) \frac{d \gamma^{j}}{d t}(t) \\
& +2\left(\gamma^{0}(t)\right)^{-1} \frac{d \gamma^{0}}{d t}(t) \frac{d \gamma^{k}}{d t}(t)=0 \text { for } 1 \leq k \leq n .
\end{aligned}
$$

Lemma 4.1. Let $\gamma(t)$ be a geodesic in $C(N)$. Then $\pi \circ \gamma(t)$ is a geodesic in $N$ whose parameter is not affine. In particular, if we change the parameter $t$ for $s(t)=c_{0} \int_{t_{0}}^{t}(\tilde{\pi} \circ \gamma(t))^{-2} d t$, with a nonzero constant $c_{0}$, then $s$ is an affine parameter of $\pi \circ \gamma$. And, if we set $c_{0}=\tilde{\pi} \circ \gamma\left(t_{0}\right) \sqrt{1-\left|\frac{d}{d t} \tilde{\pi} \circ \gamma\left(t_{0}\right)\right|^{2}}$, then $\pi \circ \gamma(s)$ has speed 1.

Proof. Let $t \rightarrow s=s(t)$ be any parameter change. Putting

$$
\begin{aligned}
\frac{d \gamma^{\alpha}}{d t}(t) & =\frac{d \gamma^{\alpha}}{d s}(s(t)) \frac{d s}{d t}(t), \\
\frac{d^{2} \gamma^{\alpha}}{d t^{2}}(t) & =\frac{d^{2} \gamma^{\alpha}}{d s^{2}}(s(t))\left\{\frac{d s}{d t}(t)\right\}+\frac{d \gamma^{\alpha}}{d s}(s(t)) \frac{d^{2} s}{d t^{2}}(t)
\end{aligned}
$$

in the equation of geodesics, we obtain 


$$
\frac{d^{2} \gamma^{k}}{d s^{2}}\left(\frac{d s}{d t}\right)+\frac{d \gamma^{k}}{d s} \frac{d^{2} s}{d t^{2}}+\sum_{i, j=1}^{n} \Gamma_{i j}^{k} \frac{d \gamma^{i}}{d s} \frac{d \gamma^{j}}{d s}\left(\frac{d s}{d t}\right)^{2}+2\left(\gamma^{0}\right)^{-1} \frac{d \gamma^{0}}{d t} \frac{d \gamma^{k}}{d s} \frac{d s}{d t}=0
$$

and we have

$$
\left(\frac{d s}{d t}\right)^{2}\left\{\frac{d^{2} \gamma^{k}}{d s^{2}}+\sum_{i, j=1}^{n} \Gamma_{i j}^{k} \frac{d \gamma^{i}}{d s} \frac{d \gamma^{i}}{d s}\right\}+\left(\frac{d \gamma^{k}}{d s}\right)\left\{\frac{d^{2} s}{d t^{2}}+2\left(\gamma^{0}\right)^{-1} \frac{d \gamma^{0}}{d t} \frac{d s}{d t}\right\}=0
$$

for $1 \leq k \leq n$. Therefore, if we take a parameter change $t \rightarrow s(t)$ which satisfies

$$
\frac{d^{2} s}{d t^{2}}+2\left(\gamma^{0}\right)^{-1} \frac{d \gamma}{d t} \frac{d s}{d t}=0
$$

then we have for $1 \leq k \leq n$

$$
\frac{d^{2} \gamma^{k}}{d s^{2}}+\sum_{i, j=1}^{n} \Gamma_{i j}^{k} \frac{d \gamma^{i}}{d s} \frac{d \gamma}{d s}=0
$$

that is, $\pi \circ \gamma(s)$ is a geodesic with affine parameter $s$. The differential equation (1) has a general solution $s(t)=c_{0} \int_{t_{0}}^{t}(\tilde{\pi} \circ \gamma(t))^{-2} d t$. In order for $\pi \circ \gamma(s)$ to have speed 1, $c_{0}$ must be chosen to be $c_{0}=\tilde{\pi} \circ \gamma\left(t_{0}\right) \sqrt{1-\left|\frac{d}{d t} \tilde{\pi} \circ \gamma\left(t_{0}\right)\right|^{2}}$.

Lemma 4.2. The length $L(\varphi)$ of the curve $\varphi$ in $C(N)$ from $x_{1}=\left(r_{1}, p_{1}\right)$ to $x_{2}=\left(r_{2}, p_{2}\right), r_{1} \leq r_{2}$, satisfies $L(\varphi) \geq r_{2}-r_{1}$.

Proof. Let $\varphi$ be parametrized so as to satisfy $\varphi(0)=x_{1}, \varphi\left(s_{0}\right)=x_{0}, \dot{\varphi}(s) \equiv 1$.

Then $L(\varphi)=s_{0}$, and

$$
s_{0}=\int_{0}^{s_{0}}\left|\frac{d}{d s} \varphi(s)\right|_{C(N)} d s \geq \int_{0}^{s_{0}} \frac{d}{d s} \tilde{\pi} \circ \varphi(s) d s=r_{2}-r_{1} .
$$

Lemma 4.3. If two geodesics $\varphi, \gamma$ in $C(N)$ satisfy

$$
\begin{gathered}
\tilde{\pi} \circ \varphi(0)=\tilde{\pi} \circ \gamma(0), \\
\frac{d}{d t} \tilde{\pi} \circ \varphi(0)>\frac{d}{d t} \tilde{\pi} \circ \gamma(0) \quad\left(\text { resp. } \frac{d}{d t} \tilde{\pi} \circ \varphi(0)=\frac{d}{d t} \tilde{\pi} \circ \gamma(0)\right),
\end{gathered}
$$

then they satisfy $\tilde{\pi} \circ \varphi(t)>\tilde{\pi} \circ \gamma(t)(\operatorname{resp} . \tilde{\pi} \circ \varphi(t)=\tilde{\pi} \circ \gamma(t))$ for $t>0$.

Proof. We have

$$
\frac{d^{2}}{d t^{2}} \tilde{\pi} \circ \gamma(t)-\left(\frac{d}{d t} \tilde{\pi} \circ \gamma(t)\right)^{-1}\left(1-\left(\frac{d}{d t} \tilde{\pi} \circ \gamma(t)\right)^{2}\right)=0
$$

From this we can see

$$
\begin{aligned}
\frac{d^{2}}{d t^{2}}\left((\tilde{\pi} \circ \gamma)^{2}\right)(t) & =2 \tilde{\pi} \circ \gamma(t) \frac{d^{2}}{d t^{2}} \tilde{\pi} \circ \gamma(t)+2\left(\frac{d}{d t} \tilde{\pi} \circ \gamma(t)\right)^{2} \\
& =2\left(1-\left(\frac{d}{d t} \tilde{\pi} \circ \gamma(t)\right)^{2}\right)+2\left(\frac{d}{d t} \tilde{\pi} \circ \gamma(t)\right)^{2} \\
& =2
\end{aligned}
$$


We have the same formulas with $\gamma$ replaced by $\varphi$. Therefore we have

$$
\frac{d}{d t}\left\{(\tilde{\pi} \circ \varphi)^{2}\right\}(t)-\frac{d}{d t}\left\{(\tilde{\pi} \circ \gamma)^{2}\right\}(t)=\frac{d}{d t}\left\{(\tilde{\pi} \circ \varphi)^{2}\right\}(0)-\frac{d}{d t}\left\{(\tilde{\pi} \circ \gamma)^{2}\right\}(0) .
$$

On the other hand,

$$
\begin{aligned}
& \frac{d}{d t}\left\{(\tilde{\pi} \circ \varphi)^{2}\right\}(0)-\frac{d}{d t}\left\{(\tilde{\pi} \circ \gamma)^{2}\right\}(0) \\
& \quad=2\left(\tilde{\pi} \circ \varphi(0) \frac{d}{d t} \tilde{\pi} \circ \varphi(0)-\tilde{\pi} \circ \gamma(0) \frac{d}{d t} \tilde{\pi} \circ \gamma(0)\right) \\
& \quad=\tilde{\pi} \circ \gamma(0)\left(\frac{d}{d t} \tilde{\pi} \circ \varphi(0)-\frac{d}{d t} \tilde{\pi} \circ \gamma(0)\right),
\end{aligned}
$$

we have, for each $t \geq 0$,

$$
\frac{d}{d t}\left((\tilde{\pi} \circ \varphi)^{2}\right)(t)-\frac{d}{d t}\left((\tilde{\pi} \circ \gamma)^{2}\right)(t)=2 \tilde{\pi} \circ \gamma(0)\left(\frac{d}{d t} \tilde{\pi} \circ \varphi(0)-\frac{d}{d t} \tilde{\pi} \circ \gamma(0)\right) .
$$

From the assumption $(\tilde{\pi} \circ \varphi(t))^{2}=(\tilde{\pi} \circ \gamma(t))^{2}$, we have for each $t \geq 0$

$$
(\tilde{\pi} \circ \varphi(t))^{2}-(\tilde{\pi} \circ \gamma(t))^{2}=t \times 2 \tilde{\pi} \circ \gamma(0)\left(\frac{d}{d t} \tilde{\pi} \circ \varphi(0)-\frac{d}{d t} \tilde{\pi} \circ \gamma(0)\right) .
$$

From this formula we can easily see that the statements of the lemma hold.

2. Proof of Proposition 3.2. Here we show that there exists a positive constant $c$ which satisfies $\{A(\partial D)\}^{n} /\{\operatorname{Vol}(D)\}^{n-1}>c$ for any domain $D$ with smooth boundary in $H_{\varepsilon} \cup C_{\varepsilon, 1}\left(S^{n-2} \times S^{1}\right)$ and for any $\varepsilon>0$.

In the following argument, we restrict $\varepsilon$ as small as we need. We fix a constant $E, E>2$.

Notations.

$$
\begin{aligned}
D_{1} & =D \cap\left(H_{\varepsilon} \cup C_{\varepsilon, E \varepsilon}\left(S^{n-2} \times S^{1}\right)\right), \\
D_{2} & =D \cap C_{E \varepsilon, 1}\left(S^{n-2} \times S^{1}\right), \\
\gamma_{1} & =\partial D \cap\left(H_{\varepsilon} \cup C_{\varepsilon, E \varepsilon}\left(S^{n-2} \times S^{1}\right)\right), \\
\gamma_{2} & =\partial D \cap C_{E \varepsilon, 1}\left(S^{n-2} \times S^{1}\right), \\
\Gamma_{\varepsilon} & =\left\{(\varepsilon, x) \in C\left(S^{n-2} \times S^{1}\right) ; x \in S^{1}\right\} \quad\left(=\partial H_{\varepsilon}\right), \\
\gamma_{\varepsilon} & =D \cap \Gamma_{\varepsilon}, \\
\widetilde{H}_{\varepsilon} & =H_{\varepsilon} \cup C_{\varepsilon, E \varepsilon}\left(S^{n-2} \times S^{1}\right) .
\end{aligned}
$$

Case 1. $\operatorname{Vol}\left(D_{1}\right) \geq \operatorname{Vol}\left(D_{2}\right)$.

In this case, since we have

$$
\begin{aligned}
& A(\partial D)\left.=A\left(\gamma_{1}\right)+A\left(\gamma_{2}\right) \geq A\left(\gamma_{1}\right)+A\left(\gamma_{E \varepsilon}\right) \quad \text { (because } D_{2} \subset C_{E \varepsilon, 1}\left(S^{n-2} \times S^{1}\right)\right) \\
&=A\left(\partial D_{1}\right) \geq\left\{\operatorname{Sob}\left(\widetilde{H}_{\varepsilon}\right)\right\}^{\frac{1}{n}}\left\{V\left(D_{1}\right)\right\}^{\frac{n-1}{n}}=\left\{\operatorname{Sob}\left(\widetilde{H}_{1}\right)\right\}^{\frac{1}{n}}\left\{V\left(D_{1}\right)\right\}^{\frac{n-1}{n}} \\
& \quad \text { (because } \widetilde{H}_{\varepsilon} \text { and } \widetilde{H}_{1} \text { are homothetic) } \\
& \geq\left(\frac{1}{2}\right)^{\frac{n-1}{n}}\left\{\operatorname{Sob}\left(\widetilde{H}_{1}\right)\right\}^{\frac{1}{n}}\{V(D)\}^{\frac{n-1}{n}},
\end{aligned}
$$

we can estimate $\{A(\partial D)\}^{n} /\{\operatorname{Vol}(D)\}^{n-1}$ from below by a positive constant independent of $\varepsilon$ and D.

Case 2. $\operatorname{Vol}\left(D_{2}\right) \geq \operatorname{Vol}\left(D_{1}\right)$.

First we give a definition and a lemma. 
Definition. For $x \in D$ we define

$$
\begin{aligned}
I_{x}=\left\{\xi \in U_{x} M_{\varepsilon}: \gamma_{\xi}(t) \in \partial D,\right. & \\
& \left.\left.\gamma_{\xi}\right|_{[0, t)} \text { is included in } D, \text { and a minimizing geodesic in } M_{\varepsilon}\right\}, \\
\omega_{x}= & \mu\left(I_{x}\right) / \mu\left(U_{x} M_{\varepsilon}\right) \quad\left(\mu \text { is the standard measure of } U_{x} M_{\varepsilon}\right) .
\end{aligned}
$$

Lemma 4.4 [Cro, p. 425]. Let the notations be as above. Then we have

$$
\frac{\{A(\partial D)\}^{n}}{\{V(D)\}^{n-1}} \geq c(n)\left\{\frac{1}{V(D)} \int_{D} \omega_{x} d V(x)\right\}^{n+1}
$$

where $c(n)$ is a positive constant depending only on the dimension $n$.

In the Case 2, using Lemma 4.4, we have

$$
\begin{aligned}
\frac{\{A(\partial D)\}^{n}}{\{V(D)\}^{n-1}} & \geq c(n)\left\{\frac{1}{V(D)} \int_{D} \omega_{x} d V(x)\right\}^{n+1} \\
& \geq c(n)\left\{\frac{1}{V(D)} \int_{D_{2}} \omega_{x} d V(x)\right\}^{n+1} \\
& \geq\left(\frac{1}{2}\right)^{n+1} c(n)\left\{\frac{1}{V\left(D_{2}\right)} \int_{D_{2}} \omega_{x} d V(x)\right\}^{n+1} \\
& \geq\left(\frac{1}{2}\right)^{n+1} c(n)\left\{\inf _{x \in D_{2}} \omega_{x}\right\}^{n+1} .
\end{aligned}
$$

Therefore we need only to estimate $\omega_{x}, x \in D_{2}$ from below.

We express $x \in D_{2}\left(\subset C\left(S^{n-2} \times S^{1}\right)\right)$ as $\left(r_{0}, p_{0}\right), r_{0} \in \mathbf{R}^{+}, p_{0} \in S^{n-2} \times S^{1}$. And we define an embedding $l: \mathbf{R}^{+} \hookrightarrow C\left(S^{n-2} \times S^{1}\right)$ by $r \mapsto\left(r, p_{0}\right)$. Let $\gamma$ be the geodesic in $M_{\varepsilon}$ which satisfies $\gamma\left(r_{0}\right)=x, \dot{\gamma}\left(r_{0}\right)=\xi$. Now we verify the following claims in order:

Claim 1. Let $\angle\left(\xi, l_{*}\left(\frac{d}{d r}\right)\right)$ be the angle at which $\xi$ and $l_{*}\left(\frac{d}{d r}\right)$ meet. If $\angle\left(\xi, l_{*}\left(\frac{d}{d r}\right)\right)<\frac{\pi}{2}$ holds, then $\gamma_{\xi}$ reaches $\Gamma_{1}$ before getting out of $C_{E \varepsilon, 1}\left(S^{n-2} \times\right.$ $\left.S^{1}\right)$.

Claim 2. Let $t_{1}\left(\geq r_{0}\right)$ be the time $\gamma_{\xi}$ reaches $\Gamma_{1}$, and $x_{1}$ be the point $\left(1, p_{1}\right)=\left(1, \pi \circ \gamma\left(t_{1}\right)\right)$. If $\angle\left(\xi, l_{*}\left(\frac{d}{d r}\right)\right)$ is small enough, then we have

$$
\operatorname{dist}_{N}\left(p_{0}, \pi \circ \gamma(t)\right)<\min \left\{\operatorname{inj}\left(S^{n-2} \times S^{1}\right), 1\right\}
$$

for each $t, r_{0} \leq t \leq t_{1}$. In particular, $\pi \circ \gamma(t), r_{0} \leq t \leq t_{1}$, is minimizing in $S^{n-2} \times S^{1}$.

Claim 3. If $\gamma$ satisfies the conclusion of Claim 2, then $\left.\gamma\right|_{\left[r_{0}, t_{1}\right]}$ is minimizing.

Proof of Claim 1. This claim can be verified from $\frac{d^{2}}{d t^{2}} \tilde{\pi} \circ \gamma>0$.

Proof of Claim 2. Using Lemma 4.1, we can express the length $s(t)$ of $\pi \circ$ $\left.\gamma\right|_{\left[r_{0}, t\right]}, t \leq r_{0}$, as

$$
s(t)=r_{0} \sqrt{1-\left|\frac{d}{d t} \tilde{\pi} \circ \gamma\left(r_{0}\right)\right|^{2}} \int_{r_{0}}^{t}(\tilde{\pi} \circ \gamma(t))^{-2} d t
$$


Now we take $\xi \in U_{x} M$ so that it satisfies $\angle\left(\xi, l_{*}\left(\frac{d}{d r}\right)\right)<\frac{\pi}{3}$. Then we have $\frac{d}{d t} \tilde{\pi} \circ \gamma\left(r_{0}\right)>\frac{1}{2}$, and from the fact that $\frac{d^{2}}{d t^{2}} \tilde{\pi} \circ \gamma>0$, we see $\frac{d}{d t} \tilde{\pi} \circ \gamma(t)>\frac{1}{2}$ for $t \geq r_{0}$. So, with the fact that $\frac{d}{d t} \tilde{\pi} \circ \gamma(t) \leq 1$, we get $\frac{t}{2} \leq \tilde{\pi} \circ \gamma(t) \leq t$ for $t \geq r_{0}$. Therefore we have

$$
\begin{aligned}
& \text { length of }\left.\pi \circ \gamma\right|_{\left[r_{0}, t\right]}=s(t) \\
& \leq r_{0} \sqrt{1-\left|\frac{d}{d t} \tilde{\pi} \circ \gamma\left(r_{0}\right)\right|^{2}} \int_{r_{0}}^{t}\left(\frac{t}{2}\right)^{-2} d t \\
& =4 r_{0} \sqrt{1-\left|\frac{d}{d t} \tilde{\pi} \circ \gamma\left(r_{0}\right)\right|^{2}}\left(r_{0}^{-1}-t^{-1}\right) \\
& \leq 4 \sqrt{1-\left|\frac{d}{d t} \tilde{\pi} \circ \gamma\left(r_{0}\right)\right|^{2}} \\
& =4 \sqrt{1-\cos ^{2} \angle\left(\xi, l_{*}\left(\frac{d}{d r}\right)\right)} \\
& =4 \sin \angle\left(\xi, l_{*}\left(\frac{d}{d r}\right)\right) \text {. }
\end{aligned}
$$

From this we see that the conclusion of Claim 2 holds if $\xi \in U_{x} M$ satisfies

$$
\angle\left(\xi, l_{*}\left(\frac{d}{d r}\right)\right)<\arcsin \left(\frac{1}{4} \min \left\{\operatorname{inj}\left(S^{n-2} \times S^{1}\right), 1\right\}\right) .
$$

Proof of Claim 3. Since $M_{\varepsilon}$ is a closed Riemannian manifold, there exists a minimizing geodesic from $x=\left(r_{0}, p_{0}\right)$ to $x_{1}=\left(1, p_{1}\right), \varphi:\left[0, t_{2}\right] \rightarrow M_{\varepsilon}, \varphi(0)$ $=x, \varphi\left(t_{2}\right)=x_{1}$. We show that $\varphi$ and $\gamma$ coincide.

Lemma 4.5. If two points $x_{3}=\left(r_{3}, p_{3}\right), x_{4}=\left(1, p_{4}\right)$, in $C_{\varepsilon, 1}\left(S^{n-2} \times S^{1}\right)$ satisfy dist $_{S^{n-2} \times S^{1}}\left(p_{3}, p_{4}\right)<1$, then we have $\operatorname{dist}_{M_{\varepsilon}}\left(x_{3}, x_{4}\right)<1$.

Proof.

$$
\begin{aligned}
\operatorname{dist}_{M_{\varepsilon}}\left(x_{3}, x_{4}\right) & \leq \operatorname{dist}_{M_{\varepsilon}}\left(\left(r_{3}, p_{3}\right),\left(r_{3}, p_{4}\right)\right)+\operatorname{dist}_{M_{\varepsilon}}\left(\left(r_{3}, p_{4}\right),\left(1, p_{4}\right)\right) \\
& \leq r_{3} \operatorname{dist}_{S^{n-2} \times S^{1}}\left(p_{3}, p_{4}\right)+\operatorname{dist}_{M_{\varepsilon}}\left(\left(r_{3}, p_{4}\right),\left(1, p_{4}\right)\right) \\
& <r_{3}+\left(1-r_{3}\right) \\
& =1
\end{aligned}
$$

Lemma 4.6. $\varphi$ is contained in $C_{\varepsilon, 2}\left(S^{n-2} \times S^{1}\right)$.

Proof. First we show that no $t \in\left[0, t_{2}\right]$ satisfies $\varphi(t) \in H_{\varepsilon}$. Suppose there exists a $t \in\left[0, t_{2}\right]$ which satisfies $\varphi(t) \in H_{\varepsilon}$. Let $t_{3}, t_{4}$ be

$$
t_{3}=\inf \left\{t \in\left[0, t_{2}\right] ; \varphi(t) \in H_{\varepsilon}\right\}, \quad t_{4}=\sup \left\{t \in\left[0, t_{2}\right] ; \varphi(t) \in H_{\varepsilon}\right\} .
$$

Then we have $L\left(\left.\varphi\right|_{\left[0, t_{2}\right]}\right) \geq L\left(\left.\varphi\right|_{\left[0, t_{3}\right]}\right)+L\left(\left.\varphi\right|_{\left[t_{4}, t_{2}\right]}\right)$, and from this inequality with Lemma 4.2 we obtain

$$
L\left(\left.\varphi\right|_{\left[0, t_{2}\right]}\right) \geq\left(r_{0}-\varepsilon\right)+(1-\varepsilon) \geq E \varepsilon-2 \varepsilon+1>1 .
$$

On the other hand, we see from Claim 2 and Lemma 4.5 (letting $x_{3}=x, x_{4}=$ $\left.x_{1}\right)$ that $x$ and $x_{1}$ satisfy the inequality $\operatorname{dist}_{M_{\varepsilon}}\left(x, x_{1}\right)<1$. But this contradicts 
the fact that $\varphi$ is minimizing. In the same way as above, we can see that no $t \in\left[0, t_{2}\right]$ satisfies $\varphi(t) \notin H_{\varepsilon} \cup C_{\varepsilon, 2}\left(S^{n-2} \times S^{1}\right)$.

From Lemma 4.6, we see that $\pi \circ \varphi(t), t \in\left[0, t_{2}\right]$ can be defined. This is a geodesic (not affinely parametrized), passing from $p_{0}$ to $p_{1}$. Now we have

$$
\int_{r_{0}}^{t_{1}} \frac{d}{d t} \tilde{\pi} \circ \gamma d t=\int_{0}^{t_{2}} \frac{d}{d t} \tilde{\pi} \circ \varphi d t=1-r_{0},
$$

since $\gamma, \varphi$ are both curves which passes from $x$ to $x_{1}$. From the fact that $\varphi$ is minimizing and that $t_{2} \leq t_{1}-r_{0}$, we obtain $\frac{d}{d t} \tilde{\pi} \circ \gamma\left(r_{0}\right) \leq \frac{d}{d t} \tilde{\pi} \circ \varphi(0)$. And, using Lemma 4.3, we have the inequality

$$
\begin{aligned}
L\left(\left.\pi \circ \varphi\right|_{\left[0, t_{2}\right]}\right) & =\tilde{\pi} \circ \varphi(0) \sqrt{1-\left|\frac{d}{d t} \tilde{\pi} \circ \varphi(0)\right|^{2}} \int_{0}^{t_{2}}(\tilde{\pi} \circ \varphi(t))^{-2} d t \\
& \leq \tilde{\pi} \circ \varphi(0) \sqrt{1-\left|\frac{d}{d t} \tilde{\pi} \circ \gamma\left(r_{0}\right)\right|^{2}} \int_{r_{0}}^{r_{0}+t_{2}}(\tilde{\pi} \circ \gamma(t))^{-2} d t \\
& \leq \tilde{\pi} \circ \gamma\left(r_{0}\right) \sqrt{1-\left|\frac{d}{d t} \tilde{\pi} \circ \gamma\left(r_{0}\right)\right|^{2}} \int_{r_{0}}^{t_{1}}(\tilde{\pi} \circ \gamma(t))^{-2} d t \\
& =L\left(\left.\pi \circ \gamma\right|_{\left[r_{0}, t_{1}\right]}\right),
\end{aligned}
$$

which is really an equality, by the fact that $\left.\pi \circ \gamma\right|_{\left[r_{0}, t_{1}\right]}$ is minimizing in $S^{n-2} \times$ $S^{1}$ (Claim 2). Therefore we have $\frac{d}{d t} \tilde{\pi} \circ \gamma\left(r_{0}\right)=\frac{d}{d t} \tilde{\pi} \circ \varphi(0), \tilde{\pi} \circ \gamma\left(r_{0}+t\right)=$ $\tilde{\pi} \circ \varphi(t), t \geq 0$, from which we can see $\pi \circ \gamma\left(r_{0}+t\right)=\pi \circ \varphi(t)$, that is, $\gamma$ and $\varphi$ coincide.

Now we complete the proof of Case 2 of Proposition 3.2. Any minimizing geodesic which starts from $x \in D_{2}$ and reaches $\Gamma_{1}$ necessarily passes $\partial D$. So, from the Claims 2 and 3, we see, for $\xi \in U_{x} M_{\varepsilon}$,

$$
\angle\left(\xi, l_{*}\left(\frac{d}{d r}\right)\right)<\arcsin \left(\frac{1}{4} \min \left\{\operatorname{inj}\left(S^{n-2} \times S^{1}\right), 1\right\}\right) \Rightarrow \xi \in I_{x} .
$$

This gives a lower estimate of $\omega_{x}=\left(\mu\left(I_{x}\right) / \mu\left(U_{x} M_{\varepsilon}\right)\right)$ independent of $\varepsilon, D$. We conclude the proof of Proposition 3.2.

\section{REFERENCES}

[A] C. Anné, Spectre du laplacien et écrasement d'anses, Ann. Sci. École Norm. Sup. (4) 20 (1987), 271-280.

[Cha-F 1] I. Chavel and E. Feldman, Spectra of manifolds with small handles, Comment. Math. Helv. 56 (1981) 83-102.

[Cha-F 2] __ Isoperimetric constants of manifolds with small handles, Math. Z. 184 (1983), 435448.

[Cha] I. Chavel, Eigenvalues in Riemannian geometry, Academic Press, 1984.

[Che] J. Cheeger, A lower bound for the smallest eigenvalue of the Laplacian, Problems in Analysis, Princeton Univ. Press, 1970, pp. 195-199.

[C-C 1] B. Colbois and G. Courtois, A note on the first nonzero eigenvalue of the Laplacian acting on p-forms, Manuscripta Math. 68 (1990), 143-160.

[C-C 2] _ Convergence de variétés et convergence du spectre du Laplacien, preprint.

[Cro] C. B. Croke, Some isoperimetric inequalities and eigenvalue estimates, Ann. Sci. Ecole Norm. Sup. Paris 13 (1980), 419-435. 
[D] J. Dodziuk, Maximal principle for parabolic inequalities and the heat flow on open manifolds, Indiana Univ. Math. J. 32 (1983), 703-716.

[F] K. Fukaya, Collapsing of Riemannian manifolds and eigenvalues of Laplace operator, Invent. Math. 87 (1987), 517-547.

Department of Mathematics, Tokyo Institute of Technology, Tokyo, Japan E-mail address: otofuji@math.titech.ac.jp 